\title{
Multivariable Prediction Model for Biochemical Response to First-Generation Somatostatin Receptor Ligands in Acromegaly
}

Eva C. Coopmans, ${ }^{1}$ Tim I.M. Korevaar, ${ }^{1}$ Sebastiaan W.F. van Meyel, ${ }^{1}$ Adrian F. Daly, ${ }^{2}$ Philippe Chanson, ${ }^{3,4}$ Thierry Brue, ${ }^{5,6}$ Brigitte Delemer, ${ }^{7}$ Václav Hána Jr., ${ }^{8}$

Annamaria Colao, ${ }^{9}$ Davide Carvalho, ${ }^{10}$ Marie-Lise Jaffrain-Rea, ${ }^{11}$ Günter K. Stalla, ${ }^{12}$ Carmen Fajardo-Montañana, ${ }^{13}$ Albert Beckers, ${ }^{2}$ Aart J. van der Lely, ${ }^{1}$

Patrick Petrossians, ${ }^{2, *}$ and Sebastian J.C.M.M. Neggers ${ }^{1, *}$

${ }^{1}$ Department of Medicine, Endocrinology section, Pituitary Center Rotterdam, Erasmus University Medical Center, Rotterdam, the Netherlands; ${ }^{2}$ Endocrinologie Centre Hospitalier Universitaire de Liège, Domaine Universitaire du Sart-Tilman, 4000, Liège, Belgium; ${ }^{3}$ Assistance Publique-Hôpitaux de Paris, Hôpital de Bicêtre, Service d'Endocrinologie et des Maladies de la Reproduction, Centre de Référence des Maladies Rares de I'Hypophyse, 94275, Le Kremlin Bicêtre, France; ${ }^{\circledR}$ Université Paris-Saclay, Univ. Paris-Sud, Inserm, Signalisation Hormonale, Physiopathologie Endocrinienne et Métabolique, 94276, Le Kremlin-Bicêtre, France; ${ }^{5}$ Aix-Marseille Université, CNRS, CRN2M UMR 7286, Marseille, France; ${ }^{6}$ APHM, Hôpital Conception, Service d'Endocrinologie, Diabète et Maladies Métaboliques, Centre de Référence des Maladies Rares d'Origine Hypophysaire, Marseille, France; ${ }^{7}$ Department of Endocrinology, Diabetes, and Nutrition, University Hospital of Reims, Reims, France; ${ }^{8} 3$ rd Department of Internal Medicine, First Medical Faculty, Charles University, Prague, Czech Republic; ${ }^{9}$ Dipartimento di Medicina Clinica e Chirurgia, Università Federico II di Napoli, Naples, Italy; ${ }^{10}$ Department of Endocrinology, Diabetes and Metabolism Section and Instituto de Investigação e Inovação em Saúde, University of Porto, Centro Hospitalar S. João, Porto, Portugal; ${ }^{11}$ Department of Biotechnological and Applied Clinical Sciences, University of L'Aquila, L'Aquila and Neuromed, IRCCS, Pozzilli, Italy; ${ }^{12}$ Clinical Neuroendocrinology, MaxPlanck-Institute of Psychiatry, Munich, Germany; and ${ }^{13}$ Servicio de Endocrinología, Hospital Universitario La Ribera, Valencia, Spain

Context: First-generation somatostatin receptor ligands (fg-SRLs) represent the mainstay of medical therapy for acromegaly, but they provide biochemical control of disease in only a subset of patients. Various pretreatment biomarkers might affect biochemical response to fg-SRLs.

Objective: To identify clinical predictors of the biochemical response to fg-SRLs monotherapy defined as biochemical response (insulin-like growth factor (IGF)-1 $\leq 1.3 \times$ ULN (upper limit of normal)), partial response ( $>20 \%$ relative IGF-1 reduction without normalization), and nonresponse ( $\leq 20 \%$ relative IGF- 1 reduction), and IGF-1 reduction.

Design: Retrospective multicenter study.

Setting: Eight participating European centers.

Methods: We performed a meta-analysis of participant data from 2 cohorts (Rotterdam and Liège acromegaly survey, 622 out of 3520 patients). Multivariable regression models were used to identify predictors of biochemical response to fg-SRL monotherapy.

Results: Lower IGF-1 concentration at baseline (odds ratio $(O R)=0.82,95 \%$ confidence interval (CI) 0.72-0.95 IGF-1 ULN, $P=.0073$ ) and lower bodyweight (OR = 0.99, 95\% Cl 0.98-0.99 kg,

ISSN Print 0021-972X ISSN Online 1945-7197

Printed in USA

C) Endocrine Society 2020. All rights reserved. For permissions, please e-mail: journals. permissions@oup.com

Received 12 June 2020. Accepted 22 June 2020.

First Published Online 26 June 2020.

Corrected and Typeset 21 July 2020
*These authors contributed equally.

Abbreviations: AIP, aryl-hydrocarbon receptor interacting protein; ATG, autogel; CV coefficient of variation; DM, diabetes; fg-SRL, first-generation somatostatin receptor ligands; $G H$, growth hormone; IGF, insulin-like growth factor; IQR, interquartile range; LAR, long-acting release; LAS, Liège Acromegaly Survey; OR, odds ratio; SE, standard error; SST2, somatostatin receptor subtype 2; ULN, upper limit of normal. 
$P=.038)$ were associated with biochemical response. Higher IGF-1 concentration at baseline (OR $=1.40$, (1.19-1.65) IGF-1 ULN, $P \leq .0001)$, the presence of type 2 diabetes (oral medication $\mathrm{OR}=2.48,(1.43-4.29), P=.0013$; insulin therapy $\mathrm{OR}=2.65,(1.02-6.70), P=.045)$, and higher bodyweight $(\mathrm{OR}=1.02,(1.01-1.04) \mathrm{kg}, P=.0023)$ were associated with achieving partial response. Younger patients at diagnosis are more likely to achieve nonresponse $(\mathrm{OR}=0.96$, (0.94-0.99) year, $P=.0070)$. Baseline IGF-1 and growth hormone concentration at diagnosis were associated with absolute IGF-1 reduction $(\beta=0.90$, standard error $(\mathrm{SE})=0.02, P \leq .0001$ and $\beta=0.002$, SE $=0.001, P=.014$, respectively).

Conclusion: Baseline IGF-1 concentration was the best predictor of biochemical response to fg$\mathrm{SRL}$, followed by bodyweight, while younger patients were more likely to achieve nonresponse. (J Clin Endocrinol Metab 105: 1-11, 2020)

Key Words. acromegaly, first-generation somatostatin receptor ligands, biochemical response

$\mathbf{F}$ irst-generation somatostatin receptor ligands (fg-SRLs) represent the mainstay of medical therapy for acromegaly $(1,2)$. Medical treatment is usually indicated for patients for whom trans-sphenoidal surgery is not an option or is not curative. Long-acting fg-SRLs act mainly by binding and activating somatostatin receptor subtype $2\left(\mathrm{SST}_{2}\right)$, which together with $\mathrm{SST}_{5}$ receptor is the most frequently expressed SST subtype of growth hormone (GH)-secreting pituitary adenomas 3-5). Overall, the efficacies of fg-SRLs, such as lanreotide autogel (ATG) and octreotide long-acting release (LAR), seem to be similar (6), and patients have biochemical response rates varying from $25 \%$ to $45 \% 6-9$ ).

Although fg-SRLs can control hormonal hypersecretion in GH-secreting pituitary adenomas, many patients are at least partially resistant to treatment and will be exposed to high $\mathrm{GH}$ and insulin-like growth factor 1 (IGF-1) concentrations. Excess of serum GH and/or IGF-1 leads to cardiovascular, metabolic, and musculoskeletal comorbidities, which, in turn, increase mortality as a result of cardiovascular, cerebrovascular, and respiratory morbidities $(10,11)$. However, mortality in patients with acromegaly significantly declined over time and could be explained by the availability of new medical treatment options $(11,12)$.

Previous studies have reported several clinical predictors of biochemical response to $\mathrm{fg}$-SRL monotherapy, including age at diagnosis, sex, bodyweight, height, serum (nadir) GH, and IGF-1 at diagnosis and baseline, tumor size, genetic mutations (eg, arylhydrocarbon receptor interacting protein), T2-weighted magnetic resonance imaging signal intensity, pathological features (granulation pattern and SSTs receptors of the adenoma) and the presence of type 2 diabetes (DM; antidiabetic medication use) as well as previous treatment modalities (surgery and medical therapy) 3-5, 13-26). Overall, these studies have used widely differing definitions of biochemical response over time and varying study entry criteria (de novo, postsurgery, or preselected sensitivity to fg-SRLs) (22). Although no validated criteria for fg-SRL therapy failure exist, only a small minority $(<10 \%)$ should be considered to be fully resistant to fg-SRLs (27). More importantly, previous studies have not been able to identify patient-specific clinical predictors for different biochemical response groups, including partial responders and nonresponders to fg-SRL therapy.

Given the importance of biochemical control in acromegaly but the lack of studies investigating predictors for the different biochemical response groups, we aim to identify clinical predictors of the biochemical response to initial treatment with the maximum dose of fg-SRLs monotherapy for at least 6 months. We categorized patients into groups by biochemical response (biochemical response, partial response, and nonresponse) and by IGF-1 reduction criteria (absolute and relative). These prediction models can be used to guide and individualize treatments and could avoid the consequences with ineffective treatment with fg-SRLs such as prolonged biochemical and metabolic disease.

\section{Patients and Methods}

\section{Cohort description}

Patients were included from 2 retrospective cohorts: (1) the Rotterdam cohort and (2) the Liège Acromegaly Survey (LAS) cohort $(21,28,29)$. The Rotterdam cohort contains data from acromegaly patients using fg-SRL monotherapy collected at the outpatient clinic of the Pituitary Center Rotterdam, Erasmus University Medical Center in Rotterdam between 1977 and 2018. The LAS cohort $(n=3194$ from 10 centers) was created using a software tool that enables hospitals throughout Europe to include acromegaly patients and report patient, biochemical, and tumor characteristics (21). For this study, only patients using fg-SRL monotherapy were enrolled from an additional 7 European centers. The inclusion period was between 1990 and 2018. Patients receiving fg-SRL monotherapy could have previously undergone surgery; however, patients that had undergone radiotherapy were excluded from the study. 
Rotterdam cohort. Clinical and biochemical data were collected from acromegaly patients initially treated with the maximum dose of fg-SRL monotherapy (ie, lanreotide ATG $120 \mathrm{mg}$ or octreotide LAR $30 \mathrm{mg}$ every 28 days) for at least 6 months. We excluded 26 patients because they were not initially treated with the maximum dose of fg-SRL monotherapy for at least 6 months. We were able to select 326 potential patients using fg-SRL monotherapy. We excluded 132 patients for 2 reasons: (1) IGF-1 normalization was achieved (defined as $\leq 1.3 \times$ upper limit of normal (ULN)) at baseline (ie, before initiating fg-SRL monotherapy, $\mathrm{n}=44$ ), and (2) follow-up data during SRL monotherapy were missing $(n=88)$. In total $(\mathrm{n}=194)$ patients remained in the cohort. The subjects then visited our outpatient clinic between every 16 and 24 weeks. At each visit to our outpatient clinic, standard measurements were performed including assessments of IGF-1 and GH concentrations. From all patients written informed consent was obtained prior to inclusion, and the study was approved by the Medical Ethics Committee of the Erasmus University Medical Center in Rotterdam.

LAS cohort. Acromegaly patients from the LAS database treated with initially the maximum dose of $\mathrm{fg}$-SRL monotherapy for at least 6 months were selected. We excluded 197 patients because they were not initially treated with the maximum dose of fg-SRL monotherapy for at least 6 months. From the LAS database, we were able to select 590 potential patients using fg-SRL monotherapy. We excluded 162 patients for 2 reasons: (1) IGF-1 normalization was achieved at baseline (defined as $\leq 1.3 \times$ ULN before initiating fg-SRL monotherapy, $n=91$ ), and follow-up data during SRL monotherapy were missing $(n=71)$. In total $(n=428)$ patients remained in the cohort. The medical ethics committee from the Liège University hospital approved the protocol and was covering the other European centers.

\section{Outcome}

The primary endpoint was the biochemical response to treatment with fg-SRLs classified as (1) biochemical response, defined as a normalized IGF-1 (IGF-1 $\leq 1.3 \times \mathrm{ULN})$ irrespective of normalized $\mathrm{GH}(\mathrm{GH} \leq 2.5 \mu \mathrm{g} / \mathrm{L})$ concentration achieved after at least 6 months of treatment; (2) partial resistance, defined as a $>20 \%$ relative reduction of IGF-1 without normalization; and (3) nonresponse, defined as a failure to decrease IGF-1 concentration by $>20 \%$, which represents the intra-assay variability. The primary endpoint was independent of $\mathrm{GH}$ values. The secondary endpoints were absolute and relative IGF-1 reduction (between serum IGF-1 at baseline versus lowest IGF-1 during fg-SRL monotherapy).

Blood measurements. In the Rotterdam cohort, total IGF-1 and GH concentrations were measured with different assays. IGF-1 concentration before and after fg-SRL treatment were analyzed using different assays: Immulite 2000 assay, a solid-phase, validated enzyme-labelled chemiluminescent immunometric assay (DPC Biermann GmbH/Siemens, Fernwald, Germany; intra-assay variability of 2-5\%, interassay variability of $3-7 \%$ ) and 2 different radioimmunoassays (Diagnostic Systems Laboratories, Webster, TX, USA, intraassay coefficient of variation (CV) $3.9 \%$, interassay CV $4.2 \%$; and Medgenix Diagnostics, Fleurus, Belgium, intra-assay CV
6.1\%, interassay CV 9.9\%). GH concentration was initially measured by immunoradiometric assay (CIS Bio International, Gif-sur-Yvette, France, intra-assay CV 2.8\%, inter-assay CV 4.4\%). Since February 2013, IGF-1 and GH concentrations were analyzed using an immunometric assay (IDS-iSYS, Boldon, UK), which is free of interference from pegvisomant. Interassay CVs for GH and IGF-1 were <5\% (GH; $n=190)$ and (IGF-1; $\mathrm{n}=190)$ in serum-based internal quality control measurements over a period of 1 year.

In the LAS cohort, containing acromegaly patients from 7 European centers, GH and IGF-1 serum measurements were assessed locally and consequently performed with different assays. In this study, the IGF-1 concentration was chosen to be expressed as the ULN of the reference ranges used in the local center (the IGF-1 divided by the age- and sex-specific ULN). $\mathrm{GH}$ concentration was measured as a single random sample and expressed as absolute value.

\section{Candidate predictors}

Variables that were considered as possible pretreatment predictors required to achieve disease control during fg-SRL monotherapy were selected based on previous studies (4, 5, $13-21,25,26,30-33)$, biological plausibility and availability of robust data ascertainment in both cohorts and included: age at diagnosis and baseline, sex, bodyweight, height, serum (nadir) GH, and IGF-1 at diagnosis and baseline, tumor size (microadenoma, macroadenoma, and nonvisible at diagnosis) and the presence of type $2 \mathrm{DM}$ at baseline as well as previous treatment modalities (surgery and medical therapy with a [partial] dopamine agonist). Bodyweight, GH, and IGF-1 concentrations were collected between 6 months before and at the time of fg-SRL initiation. Other data were collected at baseline (as indicated), were fixed data in the patient's record, or were established during the disease process.

\section{Statistical analysis}

Data are expressed as median (interquartile range [IQR]). Differences between two subgroups were analyzed using an unpaired t-test or the Mann-Whitney $U$ test (in case of a nonparametric distribution). Nominal variables were analyzed using Fisher's exact test. For all regression models, the residuals were normally distributed, final models were not affected by multicollinearity, and nonlinearity was assessed utilizing restricted cubic splines with 3 or 4 knots. We used univariable linear regression models to assess the association between each candidate predictor and the outcome. The decision for linear regression models instead of multiple models for the identification of predictors was based on Akaike information criteria and log-likelihood tests comparing multilevel models with random intercepts and/or slope per cohort versus standard linear regression correcting for cohort. To allow for optimal generalizability of effect estimates that predict the outcome, we performed standard linear regression correcting for cohort. We selected useful predictors using forward selection based on the change in regression coefficients and residual explained variability of the model, with $P<.20$ to keep predictors liberally in the model. To cope with (differentially) missing values of the candidate predictors, missing data on candidate predictors were multiple imputed (50 times). The imputation model included most candidate predictor variables (missing 
values Rotterdam/LAS cohort as percentage, bodyweight 27.8/19.9\%; height: 8.3/1.4\%; GH at diagnosis $17.5 / 11.2 \%$; tumor size $2.1 / 5.1 \%$; panhypopituitarism $0 / 69.6 \%$; age at baseline $0 / 0.5 \%$; GH at baseline $13.9 / 11.7 \%$ ) and the outcome variables. The candidate predictors age at diagnosis, gender, IGF-1 $(\times$ ULN $)$ at diagnosis and baseline, the presence of type $2 \mathrm{DM}$ and previous treatment modalities (surgery and medical therapy) had no missing values. There was no difference between the original or any of the imputed datasets. All analyses were performed in each of the completed datasets, and final results were pooled using Rubin's rules. We investigated the predictive discriminative ability of significant predictors from the multivariable analyses by performing receiver-operating-characteristics analysis. A $P$ value $<0.05$ (two-tailed) was used as a cut-off for statistical significance. All statistical analyses were performed using Statistical Package of Social Sciences, version 25.0 for Windows (SPSS) or using $\mathrm{R}$ statistical software, version 3.5.2 (packages foreign, mice, $p R O C$, and $r m s$ ).

\section{Results}

\section{Cohort characteristics}

After exclusions, the final study population comprised 662 patients (Fig. 1), the characteristics and previous treatment modalities of which are depicted in Table 1. Patients included in the LAS cohort were younger at diagnosis ( 44.0 vs 49.0 years) and more likely to be diagnosed with a macroadenoma $(81.5 \%$ vs $74.2 \%$ ). Patients from the LAS cohort more often underwent surgery and received medical treatment (ie, (partial) dopamine agonist therapy) before the start of fg-SRL and had a lower baseline IGF-1 concentration. In the total cohort the median duration of fg-SRL therapy is 132.4 months (IQR 36.4-215.3) and $284(45.7 \%)$ patients received fg-SRL as primary treatment. Observed biochemical response occurred in $80 \%$ of patients and a partial or nonsignificant response occurred in $13 \%$ and $7 \%$, respectively. No significant differences were observed in the Rotterdam and LAS cohort between excluded and included patients.

\section{Univariable predictors of the biochemical response}

All univariable analyses of the candidate predictors of biochemical response to fg-SRL monotherapy, including predictors of IGF-1 reduction, are depicted in Table 2.

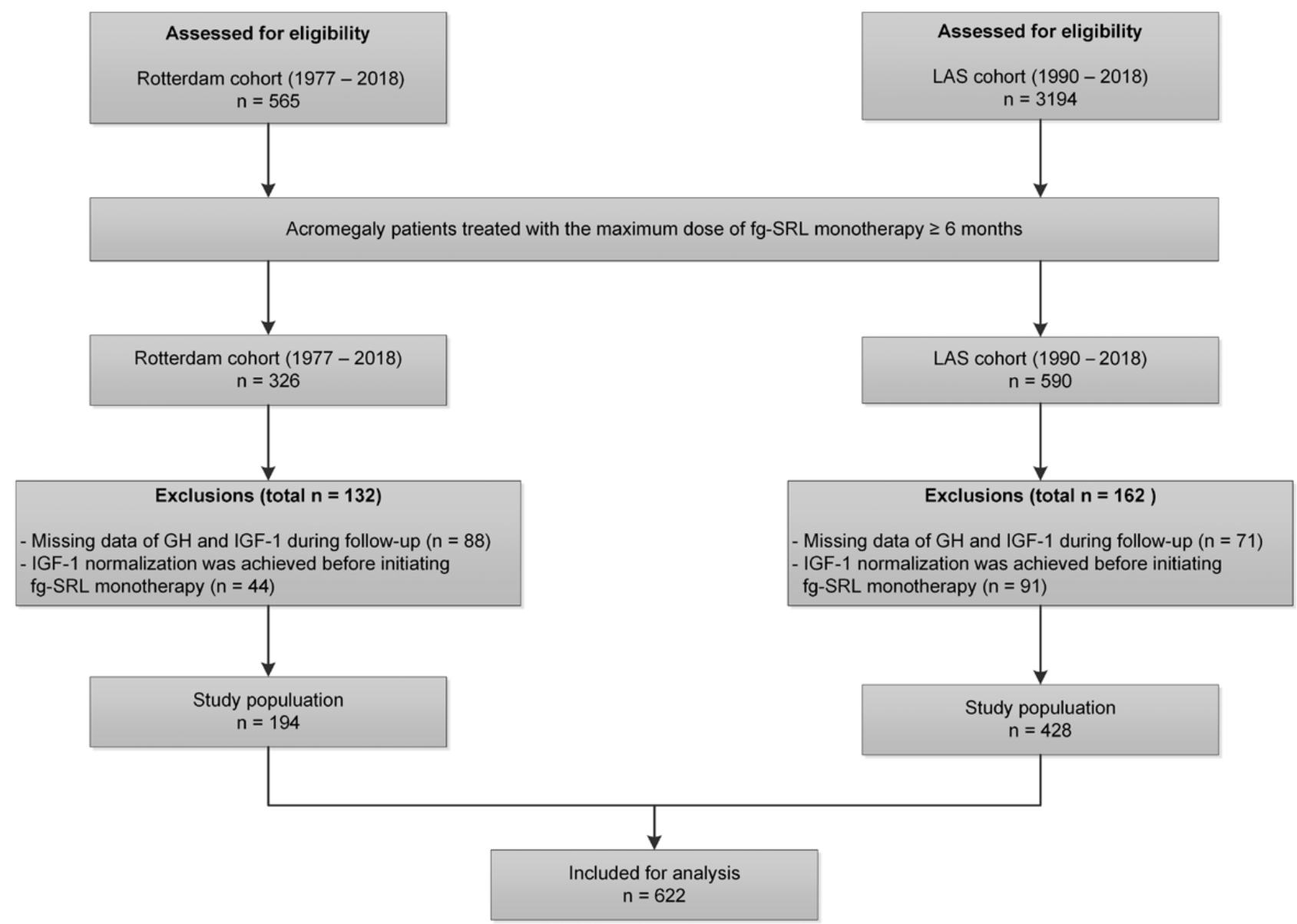

Figure 1. Flowchart of the selection procedure. IGF-1, insulin-like growth factor 1; fg-SRL, first-generation somatostatin receptor ligand; ULN, upper limit of normal. 


\begin{abstract}
Table 1. Cohort demographics and clinical characteristics of the total group, Rotterdam cohort and LAS cohort
\end{abstract}

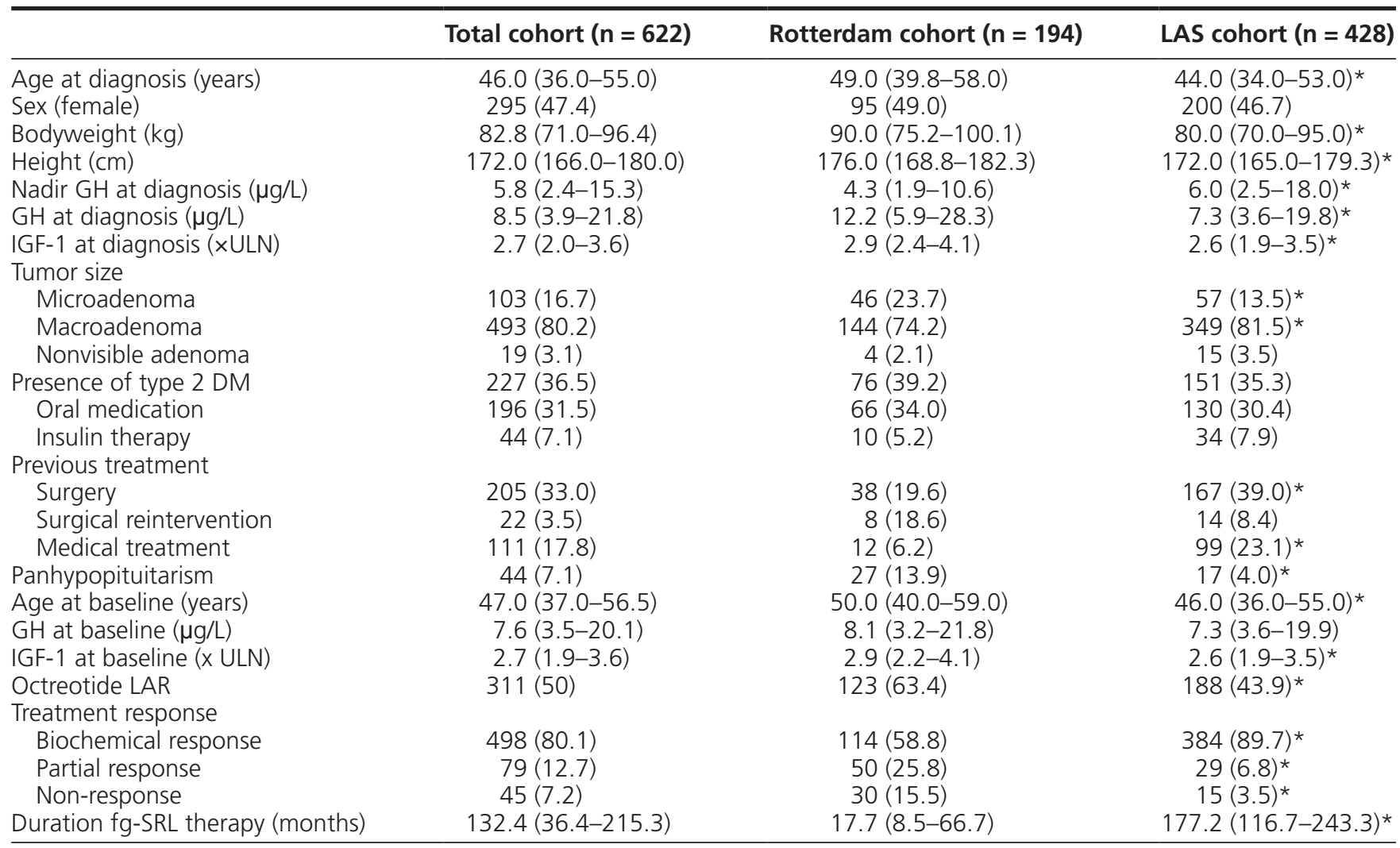

Data are mean (SD), median (IQR) or $\mathrm{n}(\%)$. Asterisk represents $P=\leq .05$ for the comparisons between Rotterdam and LAS cohort and are derived from the Student's t test (continuous variables) and Pearson's chi-square test (categorical variables).

Abbreviations: IGF-1, insulin-like growth factor 1; fg-SRL, first-generation somatostatin receptor ligand; Octreotide LAR, Octreotide long-acting release; ULN, upper limit of normal.

\section{Predictors of biochemical response}

In multivariable analyses, baseline IGF-1 concentration and bodyweight were determinants of biochemical response (Table 3). A lower IGF-1 concentration at baseline was associated with a higher chance of achieving biochemical response (OR 0.82, 95\% CI 0.72-0.95 IGF-1 ULN, $P=.0073$, Table 3). Moreover, a lower bodyweight was associated with a higher chance of a biochemical response (OR 0.99, 95\% CI 0.98-0.99 kg, $P=.038$, Table 3). The combined discriminative ability of IGF-1 concentration and bodyweight combined to predict biochemical response was adequate (AUC $0.77,95 \%$ CI 0.72-0.81; all supplementary material and figures are located in a digital research materials repository (34)).

\section{Predictors of partial response}

In the multivariable analyses, baseline IGF-1 concentration, the presence of type $2 \mathrm{DM}$ and bodyweight were determinants of partial response (Table 3). A higher IGF-1 concentration at baseline was associated with a higher chance of achieving partial response (OR 1.40, 95\% CI 1.19-1.65 IGF-1 ULN, $P \leq .0001$, Table 3). The presence of type $2 \mathrm{DM}$ was associated with a higher chance of a partial response (oral medication OR 2.48, 95\% CI 1.43-4.29, $P=.0013$; insulin therapy OR $2.65,95 \%$ CI $1.02-6.70$, $P=.045$, Table 3). Moreover, a higher bodyweight was associated with a higher chance of a partial response (OR $1.02,95 \%$ CI $1.01-1.04 \mathrm{~kg}, P=.0023$, Table 3$)$. The combined discriminative ability of the IGF- 1 concentration, the presence of type $2 \mathrm{DM}$ and bodyweight to predict partial response was good (AUC 0.80, 95\% CI 0.76-0.86 (34)).

\section{Predictors of nonresponse}

In multivariable analyses, age at diagnosis, surgery, and tumor size at diagnosis were determinants of nonresponse (Table 3). Younger patients at diagnosis had a higher chance of achieving nonresponse (OR 0.96, 95\% CI 0.940.99 year, $P=.0070$, Table 3 ). Surgery at baseline tended to be inversely associated with a higher chance of a nonresponse (OR 0.48, 95\% CI 0.22-1.05, $P=.067$, Table 3). In other words, nonresponse patients underwent surgery less often. In addition, the presence of a macroadenoma (vs microadenoma) at baseline tended to be associated with a higher chance of a nonresponse but failed to reach significance (OR 2.64, 95\% CI 0.89-7.84, $P=.081$, Table 3). Age at diagnosis, surgery and tumor size combined had 


\begin{tabular}{|c|c|c|c|c|c|}
\hline & $\begin{array}{l}\text { Biochemical } \\
\text { response }\end{array}$ & Partial response & Nonresponse & $\begin{array}{l}\text { Absolute IGF-1 } \\
\text { reduction }\end{array}$ & $\begin{array}{l}\text { Relative } \\
\text { IGF-1 re- } \\
\text { duction }\end{array}$ \\
\hline Age at diagnosis (years) & - & - & Younger* & Nonlinear* & Nonlinear ${ }^{b}$ \\
\hline Sex & - & - & - & Male* & \\
\hline Bodyweight (kg) & $\downarrow$ Bodyweight** & $\uparrow$ Bodyweight** & $\downarrow$ Bodyweight $^{b}$ & & \\
\hline $\begin{array}{l}\text { Height (cm) } \\
\text { Nadir GH at diagnosis }\end{array}$ & $\begin{array}{l}\text { Smaller* } \\
\text { Nonlinear }\end{array}$ & $\begin{array}{l}\text { Taller } \\
\text { Nonlinear* }\end{array}$ & Taller & $\begin{array}{l}\text { Nonlinear }{ }^{b} \\
\text { Nonlinear** }\end{array}$ & $\begin{array}{l}\text { Smaller**} \\
\text { Nonlinear* }\end{array}$ \\
\hline $\mathrm{GH}$ at diagnosis ( $\mu \mathrm{g} / \mathrm{L})$ & $\downarrow \mathrm{GH}^{*}$ & Nonlinear* & $\uparrow \mathrm{GH}^{\mathrm{a}}$ & Nonlinear** & - \\
\hline \multirow{2}{*}{\multicolumn{6}{|c|}{ Tumor size }} \\
\hline & & & & & \\
\hline Microadenoma & - & - & - & - & - \\
\hline Macroadenoma & - & - & Macroadenoma ${ }^{b}$ & Macroadenoma ${ }^{b}$ & - \\
\hline \multirow{2}{*}{\multicolumn{6}{|c|}{ Presence of type 2 DM }} \\
\hline & & & & & \\
\hline Oral medication & - & Yes $^{b}$ & - & - & - \\
\hline Insulin therapy & - & Yes $^{b}$ & - & - & - \\
\hline \multicolumn{6}{|l|}{ Previous treatment } \\
\hline Surgery & Yes** & Yes*** & No* & $\mathrm{No}^{* *}$ & Yes $^{\mathrm{b}}$ \\
\hline Surgical reintervention & & - & - & - & - \\
\hline Medical treatment & Yes** & Yes*** & $\mathrm{No}^{\mathrm{b}}$ & $\mathrm{No}^{* *}$ & - \\
\hline Panhypopituitarism & $\mathrm{No}^{* *}$ & Yes* & Yes** & - & $\mathrm{No}^{* *}$ \\
\hline Age at baseline (years) & & Older* & Younger* & - & - \\
\hline $\mathrm{GH}$ at baseline $(\mu \mathrm{g} / \mathrm{L})$ & Nonlinear $^{\mathrm{a}}$ & Nonlinear ${ }^{b}$ & - & $\downarrow \mathrm{GH}^{* *}$ & $\begin{array}{l}\text { Non- } \\
\text { linear** }\end{array}$ \\
\hline IGF-1 at baseline ( $x$ ULN) & $\downarrow \mid \mathrm{GF}-1 * * *$ & $\uparrow \mid \mathrm{GF}-1 * * *$ & - & $\uparrow \mid \mathrm{GF}-1 * * *$ & $\uparrow \mathrm{IGF}-1 * * *$ \\
\hline Octreotide LAR & - & - & - & - & - \\
\hline
\end{tabular}

Abbreviations: IGF-1, insulin-like growth factor 1; SRL, somatostatin receptor ligand; octreotide LAR, octreotide long-acting release; ULN, upper limit of normal.

$* P<0.05, * * P<0.01, * * * P<.0001$

${ }^{a}$ Borderline significance at $P<.07$.

${ }^{b}$ Trend towards significance at $P<.20$.

\section{Table 3. Multivariable analysis to predict biochemical, partial and non-response}

\begin{tabular}{|c|c|c|c|}
\hline & OR & $95 \% \mathrm{Cl}$ & $P$ value \\
\hline \multicolumn{4}{|l|}{ Biochemical response } \\
\hline IGF-1 at baseline (xULN) & 0.82 & $(0.72-0.95)$ & .0073 \\
\hline Bodyweight $(\mathrm{kg})$ & 0.99 & $(0.98-0.99)$ & .0379 \\
\hline \multicolumn{4}{|l|}{ Partial response } \\
\hline IGF-1 at baseline (xULN) & 1.40 & $(1.19-1.65)$ & $<.0001$ \\
\hline Type 2 DM and oral medication ${ }^{a}$ & 2.48 & $(1.43-4.29)$ & .0013 \\
\hline Type 2 DM and insulin therapy ${ }^{a}$ & 2.65 & $(1.02-6.70)$ & .0451 \\
\hline Bodyweight (kg) & 1.02 & $(1.01-1.04)$ & .0023 \\
\hline \multicolumn{4}{|l|}{ Nonresponse } \\
\hline Age at diagnosis (years) & 0.96 & $(0.94-0.99)$ & .0070 \\
\hline Surgery at baseline & 0.48 & $(0.22-1.05)$ & .0666 \\
\hline Macroadenoma $^{b}$ & 2.64 & $(0.89-7.84)$ & .0814 \\
\hline Nonvisible adenoma ${ }^{b}$ & $<0.01$ & $(<0.01-\infty)$ & .9868 \\
\hline
\end{tabular}

DM, diabetes mellitus; IGF-1, insulin-like growth hormone factor 1; SE, standard error; SRL, somatostatin receptor ligand.

${ }^{a}$ Compared with patients without type $2 \mathrm{DM}$.

${ }^{b}$ Compared with patients harboring a microadenoma.

an adequate discriminative ability to predict non-response (AUC of 0.78, 95\% CI 0.69-0.85 (34)).

\section{Predictors of IGF-1 reduction}

Multivariable analyses to predict absolute IGF-1 reduction detected a positive association for baseline IGF-1 concentration $(\beta=0.90, \mathrm{SE}=0.02, P \leq .0001$;
Fig. 2A and Table 4). A significant inverse association was seen between $\mathrm{GH}$ concentration at diagnosis and absolute IGF-1 reduction $(\beta=-0.002, \mathrm{SE}=0.001$, $P=.014$; Fig. 2B and Table 4).

With regards to relative IGF-1 reduction, the multivariable analyses include a positive association for baseline IGF-1 concentration $(\beta=6.24, \mathrm{SE}=0.66$, 
A
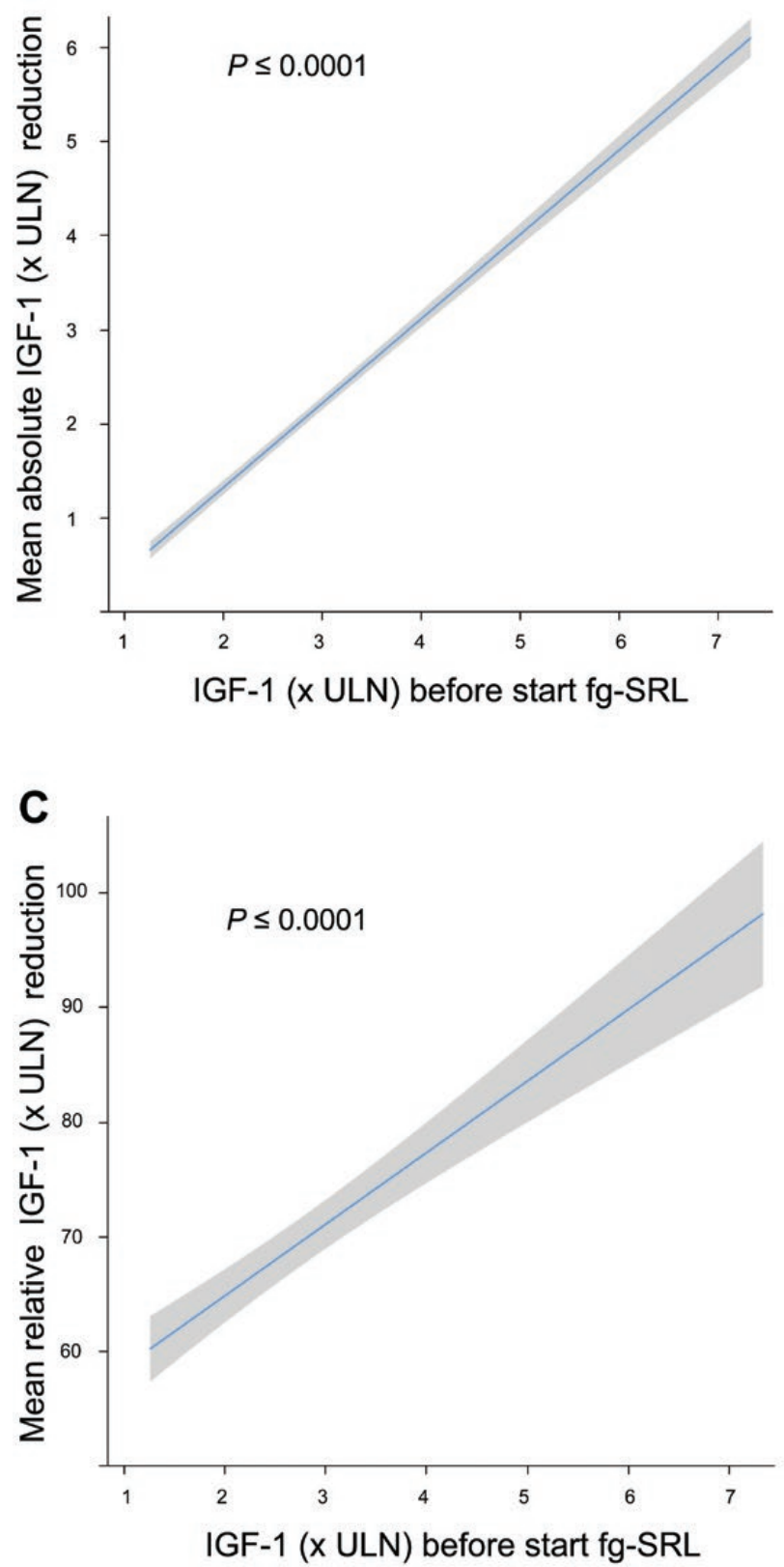

B
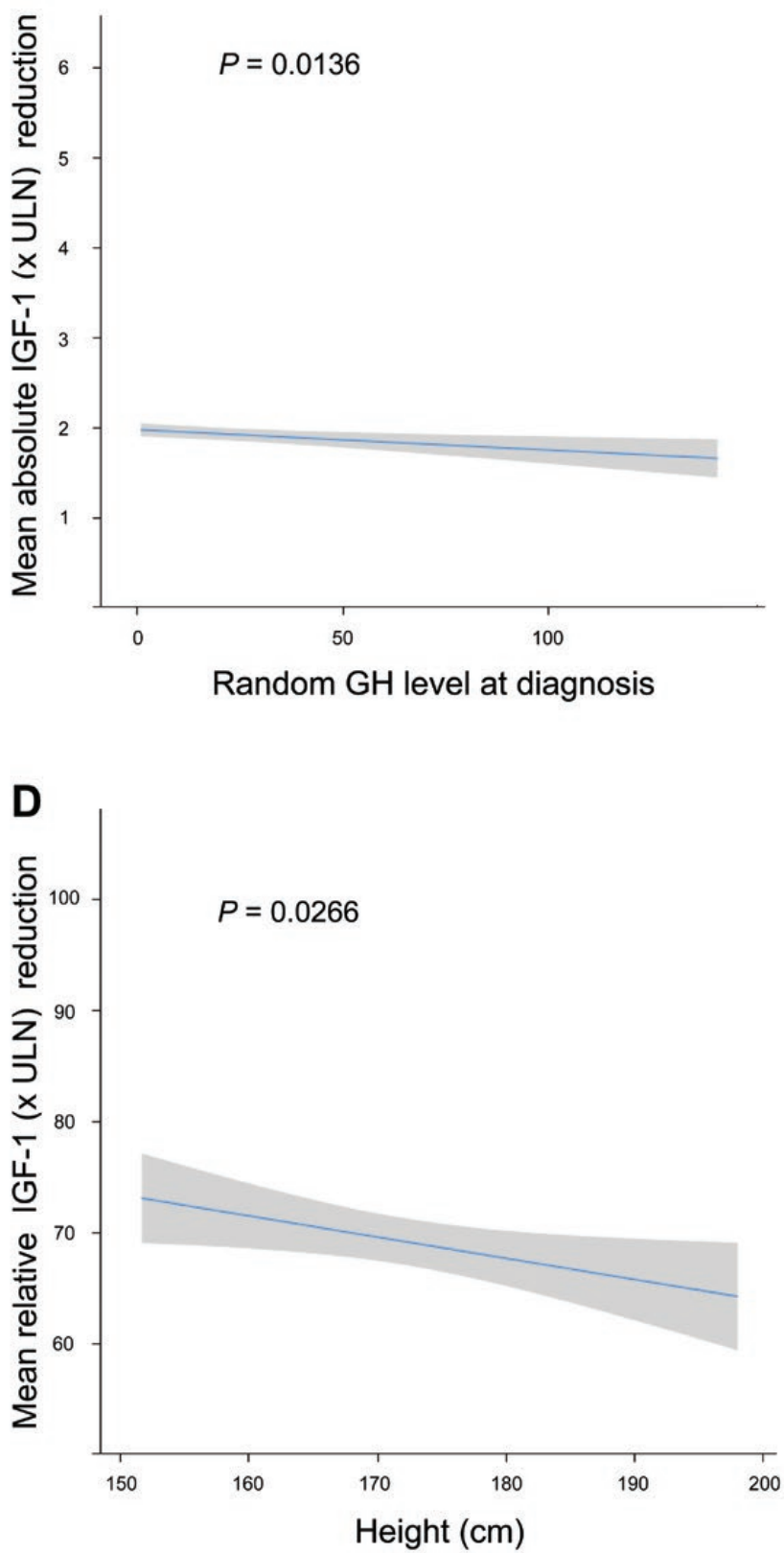

Figure 2. Predictors of IGF-1 reduction. Association between absolute IGF-1 reduction and (A) IGF-1 ( $x$ ULN) before start fg-SRL therapy and (B) GH concentration at diagnosis. Association between relative IGF-1 reduction and (C) IGF-1 ( $\times$ ULN) and (D) height before start fg-SRL therapy. The grey shade represents the $95 \%$ confidence interval of the predicted mean. IGF-1, insulin-like growth factor 1 ; fg-SRL, first-generation somatostatin receptor ligand; ULN, upper limit of normal.

Table 4. Multivariable analysis to predict IGF-1 reduction

\begin{tabular}{lccr}
\hline & $\beta$ & SE & $P$ value \\
\hline Absolute IGF-1 reduction & & & \\
IGF-1 at baseline $(\times U L N)$ & .90 & 0.02 & $<.0001$ \\
GH at diagnosis $(\mu \mathrm{g} / \mathrm{L})$ & -.002 & 0.001 & .0136 \\
Relative IGF-1 reduction & & & \\
IGF-1 at baseline $(\times U L N)$ & 6.24 & 0.66 & $<.0001$ \\
Height $(\mathrm{cm})$ & -.19 & 0.09 & .0266 \\
\hline
\end{tabular}

Abbreviations: IGF-1, insulin-like growth hormone factor 1; SE, standard error.
$P \leq .0001$; Fig. 2C and Table 4). In addition, height was inversely associated with relative IGF-1 reduction $(\beta=-0.1912, \mathrm{SE}=0.0860, P=.027$; Fig. $2 \mathrm{D}$ and Table 4$)$.

\section{Discussion}

This is the first large cohort study to focus on identifying clinical predictors of the biochemical response to treatment with fg-SRLs by categorizing patients into groups by response (biochemical response, 
partial response, and nonresponse) and by IGF-1 reduction criteria (absolute and relative). The main findings of this study are that (1) baseline IGF-1 concentration, bodyweight, and the presence of type $2 \mathrm{DM}$ can contribute to distinguishing complete biochemical from partial response and (2) that nonresponse during fg-SRLs occurred more in patients that were younger at diagnosis and tended to harbor larger tumors and underwent surgery less often.

In our study, baseline IGF-1 concentration was the best predictor of fg-SRL treatment response. A lower IGF-1 concentration at baseline was associated with a higher chance of a complete biochemical response, while a higher IGF-1 concentration was associated with a higher risk of only partial response and higher IGF-1 (absolute and relative) reduction to fg-SRLs. Previously it has been identified that lower circulating IGF-1 and $\mathrm{GH}$ levels correspond with better biochemical response to fg-SRLs $(4,21,35)$. Thus, lower baseline IGF-1 concentrations could identify patients that will achieve biochemical control, while higher baseline IGF-1 concentrations could identify those that will achieve partial responses while at the same time having a greater absolute and relative reduction in circulating IGF-1 levels. While the latter seems counterintuitive, a greater reduction even if IGF-1 levels will not decrease below the set threshold is still of clinical value in the multimodel therapy setting.

We observed that a lower bodyweight was associated with a higher chance of biochemical control, while a higher bodyweight was associated with partial response to fg-SRLs. This finding is in accordance with previous findings from the somutaline depot for acromegaly (SODA) registry (13), which identified that patients with a lower BMI $\left(\mathrm{BMI}<30 \mathrm{~kg} / \mathrm{m}^{2}\right)$ tended to have better biochemical control of IGF-1 than patients with a higher BMI $\left(\mathrm{BMI} \geq 30 \mathrm{~kg} / \mathrm{m}^{2}\right)$ after 24 months of fg-SRL therapy.

The presence of concomitant type $2 \mathrm{DM}$, besides baseline IGF-1 concentration and bodyweight, is a pretreatment predictor of partial response to fg-SRL monotherapy, is also expected. The SODA study (13), the same study as mentioned before, showed that after 24 months of fg-SRL therapy, normalization of IGF-1 concentration was achieved less frequently in diabetic patients. In a previous study of the LAS database, a significant relationship between glucose levels and IGF-1 at baseline was shown for acromegaly patients, even in the absence of type 2 DM (21). Overall, the prevalence of insulin sensitivity alterations has been found to correlate with higher BMI in patients with acromegaly as observed in the general population (36). Taking into account that type 2 DM patients likely had higher bodyweight at enrollment, both findings may be explained by the presence of hyperinsulinemia. Hyperinsulinemia may counteract possible SRLinduced pituitary-independent mechanisms of biochemical control by enhancing the synthesis of IGF-1 through upregulation of hepatic GH receptors (37, 38). Combined with the fact that fg-SRL therapy may impair glucose metabolism, largely owing to inhibition of insulin leading to further increase of glucose secretion $(39,40)$, type $2 \mathrm{DM}$ patients might benefit from initial therapy with pegvisomant instead of fg-SRLs.

Age at diagnosis appears to be the most important determinant of achieving nonresponse during fg-SRL therapy, likely reflecting (indirectly) the exposure to high circulating levels of GH and IGF-1 (18, 21, 23, $28,41)$. This observation confirms and builds upon previous studies $(21,42)$ proposing age at diagnosis to be a clinical marker of tumor size and aggressiveness. Younger patients (aged $<40$ years) tend to have larger and potentially harbor more aggressive tumors than older patients; the reason for this is unknown, although the increased prevalence of genetic mutations like AIP in younger patients with acromegaly-gigantism with fg-SRL resistance may play a role $(25,26)$.

Knowing that larger tumors secrete more GH, while older patients generally have lower GH concentrations at diagnosis $(18,21,28)$, it becomes apparent why older patients have been shown to be more sensitive to fg-SRLs therapy (18). Subsequent, surgical total tumor resection or debulking decreases the basal GH secretion, and increases the likelihood of achieving biochemical disease control with fg-SRLs. This observation is consistent with long-term trials $(20,43,44)$ and our data that show a better biochemical control of IGF-1 with fg-SRLs after surgery.

A strength of our study is the relatively large number of patients in which the biochemical responses to fg-SRL treatment were systematically investigated, while remaining to be treated with a single agent, with a stable high dose for a long period of treatment (ie, exceeding the median period of 12 months) when compared with previous literature (27). The LAS database provides some specific advantages in that it is not limited to a national dataset nor does it deal with patients managed with only a single treatment modality, and, therefore, may better reflect the general population of acromegaly patients and overcome selection bias. In our study, we utilize a more liberal definition of biochemical response (defined as IGF- $1 \leq 1.3 \times$ ULN). To overcome the limitation of a cutoff validity, we confirmed our data by using the cutoff of $\leq 1.2 \times$ ULN in the multivariable analyses, which did not affect the effect estimates of the final models. The main limitations 
of our study lie in the retrospective nature of this study. Due to the retrospective collection of data on IGF-1 concentration during fg-SRL treatment, performed on patients followed-up for at least 6 months, a selection bias cannot be ruled out. It is conceivable that patients unresponsive to fg-SRLs were switched to alternative treatment options, if available, and did not reach the 6 months follow-up period required for admission in the study. However, before considering fg-SRL resistance, at least 6 months are generally assumed to be necessary to assess treatment outcome $(19,45)$. Finally, we are limited by the use of different IGF-1 assays during fg-SRL treatment.

SRLs remain the mainstay of medical therapy in acromegaly; however, a proportion of patients are at least partially resistant to treatment. Patient-specific clinical predictors of $\mathrm{fg}$-SRL response in patients with acromegaly include baseline IGF-1 concentration as the best predictor: lower baseline IGF-1 concentrations could identify patients that will achieve biochemical control, while higher baseline IGF-1 concentrations could identify those that will achieve partial responses while at the same time having a greater absolute and relative reduction in circulating IGF-1 levels. In the latter group, a greater reduction in IGF-1 levels even it does not decrease below the set threshold is still of great clinical value in the multimodal therapy setting. Other patient-specific clinical predictors of fg-SRL response are bodyweight, the presence of type $2 \mathrm{DM}$, age at diagnosis and a trend towards tumor size and previous surgery. These prediction models can be used to guide and individualize treatments and could avoid the negative consequences of ineffective treatment with fg-SRLs such as prolonged active biochemical and metabolic disease.

\section{Acknowledgments}

The authors would like to thank all of the physicians and scientists who formed part of the "LAS Club." We thank Arash Derakshan for his help in performing the statistical analysis.

Financial Support: The Liège acromegaly survey was supported by an unrestricted educational grant from Ipsen. The study funder had no role in the collection of data, had no access to the data and had no involvement in the writing of this manuscript.

\section{Additional Information}

Correspondence and Reprint Requests: Patrick Petrossians, Endocrinologie, Centre Hospitalier Universitaire de Liège, Domaine Universitaire du Sart-Tilman, 4000, Liège, Belgium. E-mail: patrick.petrossians@chuliege.be.
Disclosure Summary: E.C. Coopmans, SW.F. van Meyel, B. Delemer, D. Carvalho, and G.K. Stalla have nothing to disclose. T.I.M. Korevaar received lecture fees from Goodlife Healthcare, Quidel, and Berlin Chemie. A.F. Daly has received grants and/or speakers fees from Pfizer and Ipsen. P. Chanson has received unrestricted research and educational grants from Ipsen, Novartis, and Pfizer as Head of the Department of Endocrinology and Reproductive Diseases, Hôpitaux Universitaires Paris-Sud. P. Chanson has served as an investigator (principal or coordinator) for clinical trials funded by Novartis, Pfizer, Ipsen, Italfarmaco, and Antisense Therapeutics. P. Chanson is a member of Advisory Boards for Ipsen and Novartis. P. Chanson has given lectures for Ipsen, Novartis, and Pfizer. All fees and honoraria are paid to his institution. V. Hána Jr. has received speaker fees and has served on Advisory Boards for Pfizer, Novartis, and Ipsen. A. Colao has been principal investigator of research studies from Novartis, Ipsen, Pfizer, and Lilly, has received research grants from Ferring, Lilly, Ipsen, Merck-Serono, Novartis, NovoNordisk, and Pfizer, has been a consultant for Novartis, Ipsen, and Pfizer, and has received fees and honoraria from Ipsen, Novartis, and Pfizer. M.L. Jaffrain-Rea has served on advisory boards for Novartis and received an educational grant from Novartis. C. Fajardo-Montañana has received honoraria from Pfizer as speaker fees. A. Beckers is a consultant for Ipsen Pharma and received research funding from Ipsen Pharma, Novartis, and Pfizer. A.J. van der Lely is a consultant to Pfizer Inc. and Millendo Therapeutics, and received grants from Pfizer Inc. P. Petrossians received speakers and consulting fees from Novartis Pharma, Ipsen Pharma, and Pfizer. S.J.C.M.M. Neggers received research grants and speaker fees from Ipsen Pharma, Novartis Pharma, Pfizer Inc., and consulting fees from Pfizer, Crinetics, and Ipsen Pharma.

Data Availability: The datasets generated during and/or analyzed during the current study are not publicly available but are available from the corresponding author on reasonable request.

\section{References}

1. Katznelson L, Laws ER Jr, Melmed S, et al.; Endocrine Society. Acromegaly: an endocrine society clinical practice guideline. $J$ Clin Endocrinol Metab. 2014;99(11):3933-3951.

2. Melmed S, Bronstein MD, Chanson P, et al. A Consensus Statement on acromegaly therapeutic outcomes. Nat Rev Endocrinol. 2018;14(9):552-561.

3. Hofland LJ, Lamberts SW. The pathophysiological consequences of somatostatin receptor internalization and resistance. Endocr Rev. 2003;24(1):28-47.

4. Cuevas-Ramos D, Fleseriu M. Somatostatin receptor ligands and resistance to treatment in pituitary adenomas. J Mol Endocrinol. 2014;52(3):R223-R240.

5. Taboada GF, Luque RM, Neto LV, et al. Quantitative analysis of somatostatin receptor subtypes (1-5) gene expression levels in somatotropinomas and correlation to in vivo hormonal and tumor volume responses to treatment with octreotide LAR. Eur J Endocrinol. 2008;158(3):295-303.

6. Murray RD, Melmed S. A critical analysis of clinically available somatostatin analog formulations for therapy of acromegaly. $J$ Clin Endocrinol Metab. 2008;93(8):2957-2968. 
7. Caron P, Petersenn S, Flanagan D, et al. Tumor responses to lanreotide autogel/depot $120 \mathrm{mg}$ after 12 and 24 weeks are correlated with tumor responses after 48 weeks in treatment-naïve acromegalic patients: post hoc analyses of the PRIMARYS study. Endocr Rev. 2014;35.

8. Melmed S, Cook D, Schopohl J, Goth MI, Lam KS, Marek J. Rapid and sustained reduction of serum growth hormone and insulin-like growth factor- 1 in patients with acromegaly receiving lanreotide autogel therapy: a randomized, placebo-controlled, multicenter study with a 52 week open extension. Pituitary. 2010;13(1):18-28.

9. Colao A, Bronstein MD, Freda P, et al.; Pasireotide C2305 Study Group. Pasireotide versus octreotide in acromegaly: a head-to-head superiority study. J Clin Endocrinol Metab. 2014;99(3):791-799.

10. Melmed S. Acromegaly pathogenesis and treatment. J Clin Invest. 2009;119(11):3189-3202.

11. Dekkers OM, Biermasz NR, Pereira AM, Romijn JA, Vandenbroucke JP. Mortality in acromegaly: a metaanalysis. J Clin Endocrinol Metab. 2008;93(1):61-67.

12. Esposito D, Ragnarsson O, Granfeldt D, Marlow T, Johannsson G, Olsson DS. Decreasing mortality and changes in treatment patterns in patients with acromegaly from a nationwide study. Eur J Endocrinol. 2018;178(5):459-469.

13. Gordon MB, Molitch ME, Woodmansee WW, et al. Body mass index as one of determinants of biochemical control in acromegaly: 2-year data from the soda registry. Endocr Rev. 2017;38(3).

14. Gadelha MR, Kasuki L, Korbonits M. Novel pathway for somatostatin analogs in patients with acromegaly. Trends Endocrinol Metab. 2013;24(5):238-246.

15. Giustina A, Mazziotti G, Torri V, Spinello M, Floriani I, Melmed S. Meta-analysis on the effects of octreotide on tumor mass in acromegaly. PLoS One. 2012;7(5):e36411.

16. Sherlock M, Fernandez-Rodriguez E, Alonso AA, et al. Medical therapy in patients with acromegaly: predictors of response and comparison of efficacy of dopamine agonists and somatostatin analogues. J Clin Endocrinol Metab. 2009;94(4):1255-1263.

17. Mazziotti G, Giustina A. Effects of lanreotide SR and Autogel on tumor mass in patients with acromegaly: a systematic review. Pituitary. 2010;13(1):60-67.

18. van der Lely AJ, Harris AG, Lamberts SW. The sensitivity of growth hormone secretion to medical treatment in acromegalic patients: influence of age and sex. Clin Endocrinol (Oxf). 1992;37(2):181-185.

19. Freda PU, Katznelson L, van der Lely AJ, Reyes CM, Zhao S, Rabinowitz D. Long-acting somatostatin analog therapy of acromegaly: a meta-analysis. J Clin Endocrinol Metab. 2005;90(8):4465-4473.

20. Petrossians P, Borges-Martins L, Espinoza C, et al. Gross total resection or debulking of pituitary adenomas improves hormonal control of acromegaly by somatostatin analogs. Eur J Endocrinol. 2005;152(1):61-66.

21. Petrossians P, Tichomirowa MA, Stevenaert A, Martin D, Daly AF, Beckers A. The Liege Acromegaly Survey (LAS): a new software tool for the study of acromegaly. Ann Endocrinol (Paris). 2012;73(3):190-201.

22. Colao A, Auriemma RS, Pivonello R, Kasuki L, Gadelha MR. Interpreting biochemical control response rates with firstgeneration somatostatin analogues in acromegaly. Pituitary. 2016;19(3):235-247.

23. Suliman M, Jenkins R, Ross R, Powell T, Battersby R, Cullen DR. Long-term treatment of acromegaly with the somatostatin analogue SR-lanreotide. J Endocrinol Invest. 1999;22(6):409-418.

24. Potorac I, Petrossians P, Daly AF, et al. T2-weighted MRI signal predicts hormone and tumor responses to somatostatin analogs in acromegaly. Endocr Relat Cancer. 2016;23(11):871-881.
25. Daly AF, Tichomirowa MA, Petrossians P, et al. Clinical characteristics and therapeutic responses in patients with germ-line AIP mutations and pituitary adenomas: an international collaborative study. J Clin Endocrinol Metab. 2010;95(11):E373-E383.

26. Beckers A, Petrossians P, Hanson J, Daly AF. The causes and consequences of pituitary gigantism. Nat Rev Endocrinol. 2018;14(12):705-720.

27. Colao A, Auriemma RS, Lombardi G, Pivonello R. Resistance to somatostatin analogs in acromegaly. Endocr Rev. 2011;32(2): 247-271.

28. Petrossians P, Daly AF, Natchev E, et al. Acromegaly at diagnosis in 3173 patients from the Liège Acromegaly Survey (LAS) database. Endocr Relat Cancer. 2017;24(10):505-518.

29. Franck SE, Korevaar TIM, Petrossians P, et al. A multivariable prediction model for pegvisomant dosing: monotherapy and in combination with long-acting somatostatin analogues. Eur J Endocrinol. 2017;176(4):421-431.

30. Gatto F, Feelders RA, van der Pas R, et al. Immunoreactivity score using an anti-sst $2 \mathrm{~A}$ receptor monoclonal antibody strongly predicts the biochemical response to adjuvant treatment with somatostatin analogs in acromegaly. J Clin Endocrinol Metab. 2013;98(1):E66-E71.

31. Hofland LJ, van der Hoek J, van Koetsveld PM, et al. The novel somatostatin analog SOM230 is a potent inhibitor of hormone release by growth hormone- and prolactin-secreting pituitary adenomas in vitro. J Clin Endocrinol Metab. 2004;89(4):1577-1585.

32. Venegas-Moreno E, Vazquez-Borrego MC, Dios E, et al. Association between dopamine and somatostatin receptor expression and pharmacological response to somatostatin analogues in acromegaly. J Cell Mol Med. 2018;22(3):1640-1649.

33. Wildemberg LE, Neto LV, Costa DF, et al. Low somatostatin receptor subtype 2, but not dopamine receptor subtype 2 expression predicts the lack of biochemical response of somatotropinomas to treatment with somatostatin analogs. J Endocrinol Invest. 2013;36(1):38-43.

34. Coopmans EC, Korevaar TIM, van Meyel SWF, et al. Multivariable prediction model for biochemical response to firstgeneration somatostatin receptor ligands in acromegaly. Figshare Repository. Deposited March 31, 2020. https://doi.org/10.6084/ m9.figshare.12053280.v2.

35. Newman CB, Melmed S, Snyder PJ, et al. Safety and efficacy of long-term octreotide therapy of acromegaly: results of a multicenter trial in 103 patients-a clinical research center study. $J$ Clin Endocrinol Metab. 1995;80(9):2768-2775.

36. Fieffe S, Morange I, Petrossians P, et al.; French Acromegaly Registry. Diabetes in acromegaly, prevalence, risk factors, and evolution: data from the French Acromegaly Registry. Eur J Endocrinol. 2011;164(6):877-884.

37. Leung KC, Doyle N, Ballesteros M, Waters MJ, Ho KK. Insulin regulation of human hepatic growth hormone receptors: divergent effects on biosynthesis and surface translocation. J Clin Endocrinol Metab. 2000;85(12):4712-4720.

38. Ciresi A, Amato MC, Pivonello R, et al. The metabolic profile in active acromegaly is gender-specific. J Clin Endocrinol Metab. 2013;98(1):E51-E59.

39. Ferone D, Colao A, van der Lely AJ, Lamberts SW. Pharmacotherapy or surgery as primary treatment for acromegaly? Drugs Aging. 2000;17(2):81-92.

40. Colao A, Ferone D, Marzullo P, et al. Long-term effects of depot long-acting somatostatin analog octreotide on hormone levels and tumor mass in acromegaly. J Clin Endocrinol Metab. 2001;86(6):2779-2786.

41. Melmed S, Braunstein GD, Horvath E, Ezrin C, Kovacs K. Pathophysiology of acromegaly. Endocr Rev. 1983;4(3):271-290.

42. Besser GM, Burman P, Daly AF. Predictors and rates of treatmentresistant tumor growth in acromegaly. Eur $J$ Endocrinol. 2005;153(2):187-193. 
43. Bevan JS, Atkin SL, Atkinson AB, et al. Primary medical therapy for acromegaly: an open, prospective, multicenter study of the effects of subcutaneous and intramuscular slow-release octreotide on growth hormone, insulin-like growth factor-I, and tumor size. J Clin Endocrinol Metab. 2002;87(10):4554-4563.

44. Colao A, Attanasio R, Pivonello R, et al. Partial surgical removal of growth hormone-secreting pituitary tumors enhances the response to somatostatin analogs in acromegaly. J Clin Endocrinol Metab. 2006;91(1):85-92.

45. Caron PJ, Bevan JS, Petersenn S, et al.; PRIMARYS Investigators. Tumor shrinkage with lanreotide autogel $120 \mathrm{mg}$ as primary therapy in acromegaly: results of a prospective multicenter clinical trial. J Clin Endocrinol Metab. 2014;99(4):1282-1290. 